\title{
Surgical selection and outcomes among elderly patients with brain arteriovenous malformations
}

\author{
Jan-Karl Burkhardt, MD, ${ }^{1}$ Ethan A. Winkler, MD, PhD, ${ }^{2}$ Joshua S. Catapano, MD, ${ }^{3}$ \\ Robert F. Spetzler, MD, ${ }^{3}$ and Michael T. Lawton, MD $^{3}$
}

\begin{abstract}
${ }^{1}$ Department of Neurosurgery, Baylor College of Medicine Medical Center, Houston, Texas; 'Department of Neurological Surgery, University of California, San Francisco, California; and ' ${ }^{2}$ epartment of Neurosurgery, Barrow Neurological Institute, St. Joseph's Hospital and Medical Center, Phoenix, Arizona
\end{abstract}

\begin{abstract}
OBJECTIVE Studies of resection of brain arteriovenous malformations (AVMs) in the elderly population are scarce. This study examined factors influencing patient selection and surgical outcome among elderly patients.

METHODS Patients 65 years of age and older who underwent resection of an unruptured or ruptured brain AVM treated by two surgeons at two centers were identified. Patient demographic characteristics, AVM characteristics, clinical presentation, and outcomes measured using the modified Rankin Scale (mRS) were analyzed. For subgroup analyses, patients were dichotomized into two age groups (group 1, 65-69 years old; group 2, $\geq 70$ years old).

RESULTS Overall, 112 patients were included in this study (group 1, $n=61$; group 2, $n=51$ ). Most of the patients presented with hemorrhage (71\%), a small nidus (<3 cm, 79\%), and a low Spetzler-Martin (SM) grade (grade I or II, 63\%) and were favorable surgical candidates according to the supplemented SM grade (supplemented SM grade $<7,79 \%$ ). A smaller AVM nidus was statistically significantly more likely to be present in patients with infratentorial AVMs $(p=0.006)$ and with a compact AVM nidus structure $(p=0.02)$. A larger AVM nidus was more likely to be treated with preoperative embolization ( $p$ 0.001). Overall outcome was favorable (mRS scores $0-3$ ) in $71 \%$ of the patients and was statistically independent from age group or AVM grading. Patients with ruptured AVMs at presentation had significantly better preoperative mRS scores $(p<0.001)$ and more favorable mRS scores at the last follow-up $(p=0.04)$ than patients with unruptured AVMs.
\end{abstract}

CONCLUSIONS Outcomes were favorable after AVM resection in both groups of patients. Elderly patients with brain AVMs treated microsurgically were notable for small nidus size, AVM rupture, and low SM grades. Microsurgical resection is an important treatment modality for elderly patients with AVMs, and supplemented SM grading is a useful tool for the selection of patients who are most likely to achieve good neurological outcomes after resection.

https://thejns.org/doi/abs/10.3171/2020.7.FOCUS20464

KEYWORDS arteriovenous malformation; AVM; elderly; microsurgical resection; supplemented Spetzler-Martin grading

$\mathrm{B}$ RAIN arteriovenous malformations (AVMs) are rare pathological tangles of blood vessels forming a nidus without intervening capillaries and with highpressure shunting between feeding arteries and draining veins. ${ }^{1,2}$ Most patients with AVMs receive a diagnosis early in life, and treatment decisions depend on different factors including the location and size of the AVM, whether there is hemorrhage at presentation, the venous draining pattern, the compactness of the AVM nidus, and patient age. ${ }^{3}$ It is known that the presence of hemorrhage is an important factor, and patients with hemorrhage have a significantly higher risk of rehemorrhage if the AVM is left untreated. ${ }^{3,4}$ All of these factors are weighed against the lifetime risk of hemorrhage, and common grading systems, such as the Spetzler-Martin (SM), Lawton-Young (LY), and combination (supplemented SM [supp-SM]) grading systems, incorporate these factors to identify surgical candidates and predict risks of operative morbidity. ${ }_{5,6}$

ABBREVIATIONS AVM = arteriovenous malformation; BNI = Barrow Neurological Institute; LY = Lawton-Young; mRS = modified Rankin Scale; SM = Spetzler-Martin; supp-SM = supplemented SM; UCSF = University of California, San Francisco.

SUBMITTED May 29, 2020. ACCEPTED July 17, 2020.

INCLUDE WHEN CITING DOI: 10.3171/2020.7.FOCUS20464. 
Although it is uncommon for AVMs to be first diagnosed late in life, such cases are possible. ${ }^{7-10}$ Making a decision regarding AVM treatment for elderly patients is challenging due to the limited capacity for neurological recovery and increased general risk associated with surgery among this population, compared with younger patients. Supp-SM grading incorporates patient age into the scoring system and adds 3 points to the score for every AVM patient who is older than 40 years to adjust for this risk. ${ }^{5,11}$ Therefore, elderly patients are frequently moved into the intermediate grades of this grading system (suppSM grades 5, 6, and 7). A limit of operability is set crudely at less than or equal to supp-SM grade 6, which can affect the selection of elderly patients for brain AVM surgery. Specifically, advanced age may skew patient selection toward AVMs with lower SM grades to fit below operability limits, selecting patients with superficial and noneloquent lesions or patients who present with hemorrhage. Conversely, patients with high SM grades or AVMs without evidence of hemorrhage may be preferentially observed. In this report, the largest in the published literature, we examine surgical selection among elderly patients with AVMs treated by two surgeons (R.F.S. and M.T.L.) at two institutions (Barrow Neurological Institute, St. Joseph's Hospital and Medical Center, Phoenix, Arizona [BNI], and University of California, San Francisco [UCSF], respectively) and the outcomes associated with these decisions in an effort to better characterize proper decisionmaking in this patient population.

\section{Methods \\ Study Design}

This study was approved by the institutional review boards of both institutions and was performed in compliance with Health Insurance Portability and Accountability Act regulations. All operations were performed by the senior authors (M.T.L. and R.F.S.) between 1997 and 2019. Patients 65 years of age or older who had undergone resection of AVMs were identified from our prospective databases. In addition to demographic characteristics, AVM-specific factors, such as grading systems (SM and supp-SM), symptoms at presentation, ruptured or unruptured AVM status before surgery, AVM-associated aneurysms, the use of preoperative endovascular embolization or radiosurgery, and outcomes measured by the modified Rankin Scale (mRS), were collected from the database. For subgroup analyses, patients were stratified into two age groups: 65-69 years old (group 1) and at least 70 years old (group 2). Patients missing AVM-specific information, demographic characteristics, or outcome data and patients for whom histology results did not confirm a brain AVM were excluded from this study.

\section{Statistical Analysis}

Statistical analysis was performed using IBM SPSS (version 22, IBM Corp.), and figures were generated using Excel (version 14.6, Microsoft Corp.). Continuous variables are presented as means and standard errors. Comparisons between groups were performed using the Mann-Whitney U-test for continuous parameters and the chi-square test or Fisher exact test for categorical parameters. Statistical significance was set at $\mathrm{p}<0.05$.

\section{Results}

Overall, 112 elderly patients with brain AVMs who met the inclusion criteria were identified, with similar distribution between the two participating centers (UCSF, $n$ $=63$; BNI, $\mathrm{n}=49$ ). Most of the patients presented with hemorrhage ( 80 of $112,71 \%$ ), a small nidus ( $<3 \mathrm{~cm}$; 88 of $112,79 \%$ ), and low SM grade (grades I and II; 70 of 112 , 63\%). Most patients had acceptable surgical risk, with a supp-SM grade of 6 or lower (89 of 112, 79\%) (Tables 1 and 2). Among patients with a small AVM size, there was no statistically significant difference between those who presented with ruptured versus unruptured AVMs ( $\mathrm{p}=$ 0.80 ). In addition to hemorrhage, neurological deficits (43 of $112,38 \%$ ) were the most common presenting symptom. Twenty-nine (26\%) of the patients received an incidental diagnosis (Table 1). Incidental AVMs were more likely to be high-grade AVMs (SM grade I or II: 10 of 29, 34\%; SM grade III-V: 19 of 29,66\%), but the distribution of lowgrade versus high-grade AVMs was comparable to that for symptomatic AVMs ( $p=0.83$ ).

Patients were then grouped by age (group 1: 65-69 years old, $\mathrm{n}=61$; group $2: \geq 70$ years old, $\mathrm{n}=51$ ) with a mean age of 67.0 years (group 1) and 75.5 years (group 2). There was no statistically significant difference between the two age groups in terms of where they were treated, sex, clinical presentation, AVM size, SM grade, LY grade, supp-SM grade, AVM location, or infratentorial location $(p \geq 0.15)$ (Table 1$)$. There were significantly more AVMassociated aneurysms in group 1 than in group 2 (21 vs 9 , $\mathrm{p}=0.046$ ), and preoperative embolization was more likely to be performed in group 1 ( $31 \mathrm{vs} 15, \mathrm{p}=0.02)$. There was no difference in AVM nidus size between the two groups. Patients with infratentorial AVMs $(p=0.006)$ and a more compact nidus structure $(\mathrm{p}=0.02)$ had AVMs that were statistically significantly smaller compared with patients with supratentorial AVMs and those with diffuse nidus structure, respectively (Fig. 1A and B). Patients who underwent preoperative embolization had significantly larger AVMs than patients without preoperative embolization (p < 0.001) (Fig. 1C).

Overall outcome at last follow-up (mean follow-up, 12.1 months; range 0.1-91.7 months) was favorable (mRS scores $0-3)$ in 79 of $112(71 \%)$ patients and good (mRS score $0-2$ ) in 56 of $112(50 \%)$ patients (Table 2 and Fig. 2). Overall, neurological condition was significantly improved compared with the preoperative status for patients overall $(p<0.001)$ as well as for each of the two age groups separately (Fig. 2A-D). A preoperative mRS score of 0-3 was present in 64\% (72 of 112), and a score of 0-2 was present in 45\% (50 of 112). There was no difference between preoperative or postoperative mRS score between age groups, with a comparable follow-up time (14.8 months vs 8.9 months, $\mathrm{p}=0.20$ ). Patients were also stratified by low versus high SM grade or LY grade, and the outcome measured by preoperative and postoperative mRS score was comparable for both age groups (Table 2). Patients with ruptured AVMs at presentation showed a significantly better preoperative mRS score $(\mathrm{p}<0.001)$ 
TABLE 1. Clinical and radiological characteristics of patients with AVM stratified by age

\begin{tabular}{|c|c|c|c|c|}
\hline Characteristic & All Patients $(n=112)$ & Age $65-69$ Yrs $(n=61)$ & Age $\geq 70$ Yrs $(n=51)$ & $\mathrm{p}$ Value \\
\hline Sex & & & & 0.70 \\
\hline Male & $61(54)$ & $32(52)$ & $29(57)$ & \\
\hline Female & $51(46)$ & $29(48)$ & $22(43)$ & \\
\hline Age in yrs, mean (range) & $70.9(65-90)$ & $67.0(65-69)$ & $75.5(70-90)$ & $<0.001$ \\
\hline Treatment center & & & & 0.34 \\
\hline UCSF & $63(56)$ & $37(61)$ & $26(51)$ & \\
\hline $\mathrm{BNI}$ & $49(44)$ & $24(39)$ & $25(49)$ & \\
\hline Clinical presentation & & & & 0.25 \\
\hline Seizure & $11(10)$ & $6(10)$ & $5(10)$ & \\
\hline Headache & $22(20)$ & $13(21)$ & $9(18)$ & \\
\hline Neurological deficits & $43(38)$ & $18(30)$ & $25(49)$ & \\
\hline Incidental & $29(26)$ & $20(33)$ & $9(18)$ & \\
\hline Other & $7(6)$ & $4(7)$ & $3(6)$ & \\
\hline Hemorrhage, n (\%) & $80(71)$ & $40(66)$ & $40(78)$ & 0.15 \\
\hline AVM diameter in cm, mean (range) & $2.3(0.4-9.4)$ & $2.6(0.7-9.4)$ & $2(0.4-6)$ & 0.08 \\
\hline AVM diameter in $\mathrm{cm}$ & & & & 0.15 \\
\hline$<3$ & $88(79)$ & $43(70)$ & $45(88)$ & \\
\hline $3-6$ & $21(19)$ & $16(26)$ & $5(10)$ & \\
\hline$>6$ & $3(3)$ & $2(3)$ & $1(2)$ & \\
\hline Deep venous drainage & $50(45)$ & $23(38)$ & $27(53)$ & 0.13 \\
\hline Eloquence & $58(52)$ & $27(44)$ & $31(61)$ & 0.09 \\
\hline AVM side & & & & 0.32 \\
\hline Right & $45(40)$ & $22(36)$ & $23(45)$ & \\
\hline Left & $56(50)$ & $32(52)$ & $24(47)$ & \\
\hline Midline & $11(10)$ & $7(11)$ & $4(8)$ & \\
\hline SM grade & & & & 0.38 \\
\hline 1 & $27(24)$ & $16(26)$ & $11(22)$ & \\
\hline II & $43(38)$ & $26(43)$ & $17(33)$ & \\
\hline III & $32(29)$ & $13(21)$ & $19(37)$ & \\
\hline IV & $9(8)$ & $5(8)$ & $4(8)$ & \\
\hline V & $1(1)$ & $1(2)$ & $0(0)$ & \\
\hline Nidus compact & $93(83)$ & $49(80)$ & $44(86)$ & 0.46 \\
\hline Associated aneurysms & $30(27)$ & $21(34)$ & $9(18)$ & 0.046 \\
\hline Infratentorial location & $43(38)$ & $22(36)$ & $21(41)$ & 0.70 \\
\hline LY grade & & & & 0.20 \\
\hline III & $66(59)$ & $33(54)$ & $33(65)$ & \\
\hline IV & $43(38)$ & $25(41)$ & $18(35)$ & \\
\hline V & $3(3)$ & $3(5)$ & $0(0)$ & \\
\hline Preop embolization & $46(41)$ & $31(51)$ & $15(29)$ & 0.02 \\
\hline Previous radiosurgery & $6(5)$ & $3(5)$ & $3(6)$ & $>0.99$ \\
\hline
\end{tabular}

Data are number (\%) of patients unless otherwise indicated. Boldface type indicates statistical significance.

as well as a more favorable mRS score at last follow-up $(p=0.04)$. This favorable effect of surgical treatment was also observed for patients who presented with hemorrhage stratified by age group $(\mathrm{p} \leq 0.006)$ but not for patients who presented without hemorrhage ( $\mathrm{p} \geq 0.06$ ) (Fig. $2 \mathrm{E}$ and $\mathrm{F}$ ).

\section{Discussion}

This study represents the largest cohort of elderly pa- tients with brain AVMs treated microsurgically, and we found favorable postoperative outcomes for patients in both age groups. We identified factors influencing decision-making in microsurgical resection of brain AVMs among the elderly population, namely, hemorrhagic presentation and small AVM size. These are factors that are assigned 0 points and 1 point in the LY and SM grading systems, respectively, and elderly patients with these fac- 
TABLE 2. Outcomes among patients with AVM stratified by SM and supp-SM grades

\begin{tabular}{|c|c|c|c|c|}
\hline Outcome & $\begin{array}{c}\text { All } \\
\text { Patients }\end{array}$ & $\begin{array}{c}\text { Age } \\
65-69 \text { Yrs }\end{array}$ & $\begin{array}{c}\text { Age } \\
\geq 70 \text { Yrs }\end{array}$ & $p$ Value \\
\hline All grades & $n=112$ & $n=61$ & $\mathrm{n}=51$ & \\
\hline Preop mRS score & & & & 0.18 \\
\hline $0-2$ & $50(45)$ & $31(51)$ & $19(37)$ & \\
\hline $3-6$ & $62(55)$ & $30(49)$ & $32(63)$ & \\
\hline Postop mRS score & & & & 0.71 \\
\hline $0-2$ & $56(50)$ & $32(52)$ & $24(47)$ & \\
\hline $3-6$ & $56(50)$ & $29(48)$ & $27(53)$ & \\
\hline SM grade $\leq I I$ & $n=70$ & $\mathrm{n}=42$ & $n=28$ & \\
\hline Preop mRS score & & & & $>0.99$ \\
\hline $0-2$ & $36(51)$ & $22(52)$ & $14(50)$ & \\
\hline $3-6$ & $34(49)$ & $20(48)$ & $14(50)$ & \\
\hline Postop mRS score & & & & $>0.99$ \\
\hline $0-2$ & $41(59)$ & $25(60)$ & $16(57)$ & \\
\hline $3-6$ & $29(41)$ & $17(40)$ & $12(43)$ & \\
\hline SM grade $\geq|I|$ & $n=42$ & $n=19$ & $n=23$ & \\
\hline Preop mRS score & & & & 0.11 \\
\hline $0-2$ & $14(33)$ & $9(47)$ & $5(22)$ & \\
\hline $3-6$ & $28(67)$ & $10(53)$ & $18(78)$ & \\
\hline Postop mRS score & & & & $>0.99$ \\
\hline $0-2$ & $15(36)$ & $7(37)$ & $8(35)$ & \\
\hline $3-6$ & $27(64)$ & $12(63)$ & $15(65)$ & \\
\hline Supp-SM grade $\leq 6$ & $\mathrm{n}=89$ & $n=49$ & $n=40$ & \\
\hline Preop mRS score & & & & 0.52 \\
\hline $0-2$ & $40(45)$ & $24(49)$ & $16(40)$ & \\
\hline $3-6$ & $49(55)$ & $25(51)$ & $24(60)$ & \\
\hline Postop mRS score & & & & 0.53 \\
\hline $0-2$ & $48(54)$ & $28(57)$ & $20(50)$ & \\
\hline $3-6$ & $41(46)$ & $21(43)$ & $20(50)$ & \\
\hline Supp-SM grade >6 & $n=23$ & $n=12$ & $\mathrm{n}=11$ & \\
\hline Preop mRS score & & & & 0.21 \\
\hline $0-2$ & $10(43)$ & $7(58)$ & $3(27)$ & \\
\hline $3-6$ & $13(57)$ & $5(42)$ & $8(73)$ & \\
\hline Postop mRS score & & & & $>0.99$ \\
\hline $0-2$ & $8(35)$ & $4(33)$ & $4(36)$ & \\
\hline $3-6$ & $15(65)$ & $8(67)$ & $7(64)$ & \\
\hline
\end{tabular}

Data are number (\%) of patients.

tors may still be operable according to the supp-SM grade cutoff. Indeed, we identified low grades based on the SM and supp-SM grading systems as significant factors influencing patient selection. Outcomes were comparable for both age groups, but patients with ruptured AVMs at presentation had significantly better outcomes than patients with other presenting symptoms.

These findings confirm the results of previously published studies of AVMs among the elderly population that demonstrated an important role for surgery in achieving favorable outcomes. ${ }^{8,9,12}$ It has been shown that the likelihood of hemorrhage is higher among patients with ad- vanced age than among younger patients. ${ }^{1}$ In our earlier publication, we were also able to show that the prevalence of hemorrhagic presentation is high (49\%) among patients with AVMs who are older than 60 years. ${ }^{8}$ Results of these single-center studies were recently confirmed by a large meta-analysis of more than 2000 patients and more than 6000 patient-years that confirmed the increasing risk of AVM rupture with increasing age. ${ }^{13}$ Our previously published monocentric study compared patients treated microsurgically with those treated medically in two age groups (60-65 years and $>65$ years), ${ }^{8}$ whereas this bicentric study focused on microsurgically treated patients only who were older than 65 years. Consequently, the number of microsurgical patients $>65$ years of age in the current study was nearly three times that of the prior study.

Interestingly, hemorrhage does not seem to be a predictor of unfavorable outcomes among elderly patients with AVMs. As shown in our patient cohort, patients who presented with hemorrhage had a significantly better outcome than patients who did not present with hemorrhage. Similar findings were reported by Lanzino et al. in their study involving patients older than 60 years with intraparenchymal hemorrhage due to a ruptured AVM. ${ }^{9}$ They showed that 8 of 13 patients survived and that 6 of the 8 surviving patients regained independent activity. This phenomenon may be explained by the fact that the hemorrhage causes deficits by mass effect rather than by direct parenchymal damage, and that recovery is possible after hematoma evacuation and AVM resection. Also, hematoma cavities minimize brain transgression with surgery and make resection more favorable, as shown in the LY score. ${ }^{5}$ Therefore, patients who present with hemorrhage may have lower surgical risk than patients with unruptured AVMs., ${ }^{5,14}$

Small AVM size was common in our patient cohort, with an AVM diameter of less than $3 \mathrm{~cm}$ in $79 \%$ of our patients. Small AVM size may also explain the high rate of hemorrhage in our patient cohort. Previous studies have shown that patients with smaller AVMs are more likely to experience hemorrhage than patients with larger AVMs. ${ }^{15}$ Another theory regarding why older patients with AVMs may be more likely to present with smaller AVMs is that patients with smaller AVMs remain free of symptoms until a later age, compared with patients with larger AVMs. Smaller AVMs may be less likely to cause steal-related neurological deficits or seizures. Hashimoto et al. reported that all of their elderly patients with resected AVMs had AVMs that were less than $4 \mathrm{~cm}$ in diameter. ${ }^{12}$ Smaller AVMs are also more favorable for surgery and are overrepresented among this elderly surgical population due to the artifacts and biases introduced by supp-SM grading. Elderly patients are assigned 3 points for age in the suppSM grading system, and with cutoffs for operability set at supp-SM grade 6, there is little room for additional points for deep venous drainage, eloquence, diffuseness, and unruptured presentation. As a result, the application of suppSM grading skews patient selection toward smaller AVMs. The good outcomes observed in this experience confirm the utility of supp-SM grading for patient selection. Patients with larger, higher-grade AVMs were not selected for surgery in this study and might have compromised the outcome results. These results support a more conserva- 

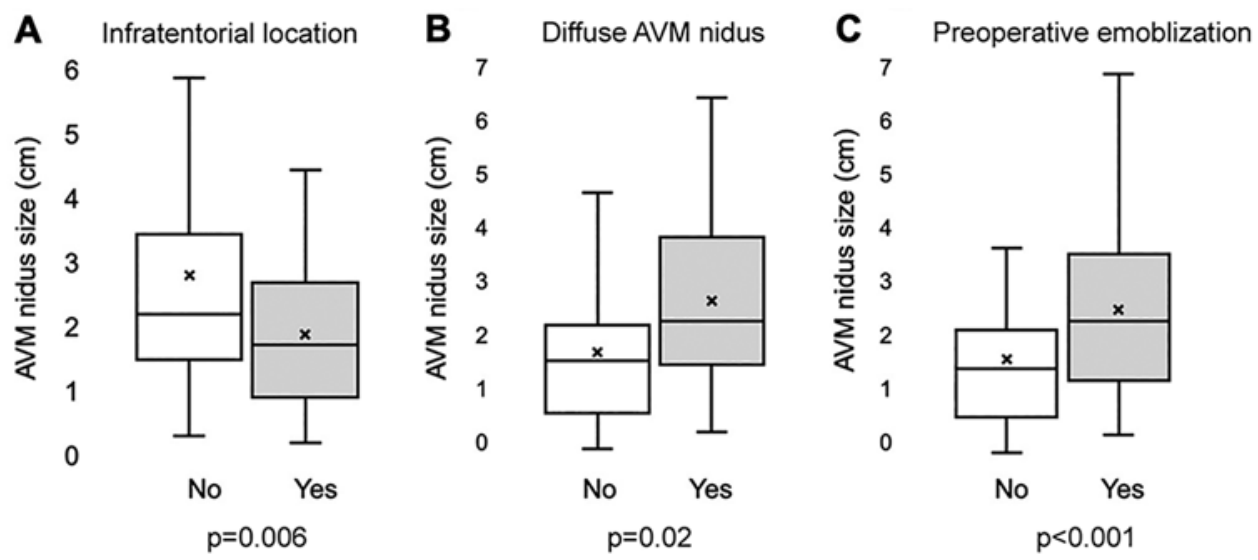

FIG. 1. Boxplot analysis of AVM nidus size in relationship to infratentorial AVM location (A), diffuse nidus structure (B), and preoperative embolization (C). The top and bottom horizontal boundaries of the boxes indicate the 75th and 25th percentiles, respectively; the horizontal line indicates the median value; the $x$ indicates the mean value; and the whiskers above and below indicate the 90 th and 10 th percentiles, respectively.

tive selection posture guided by supp-SM grading in this elderly patient population.

Comorbidities and frailty of the elderly patients were not analyzed with specific metrics. However, the overall health of the elderly AVM patients was accounted for indirectly in the selection of patients for surgery, with those having multiple severe comorbidities generally not selected for surgery by the senior surgeons.

\section{Conclusions}

In this study, we were able to show that outcomes are
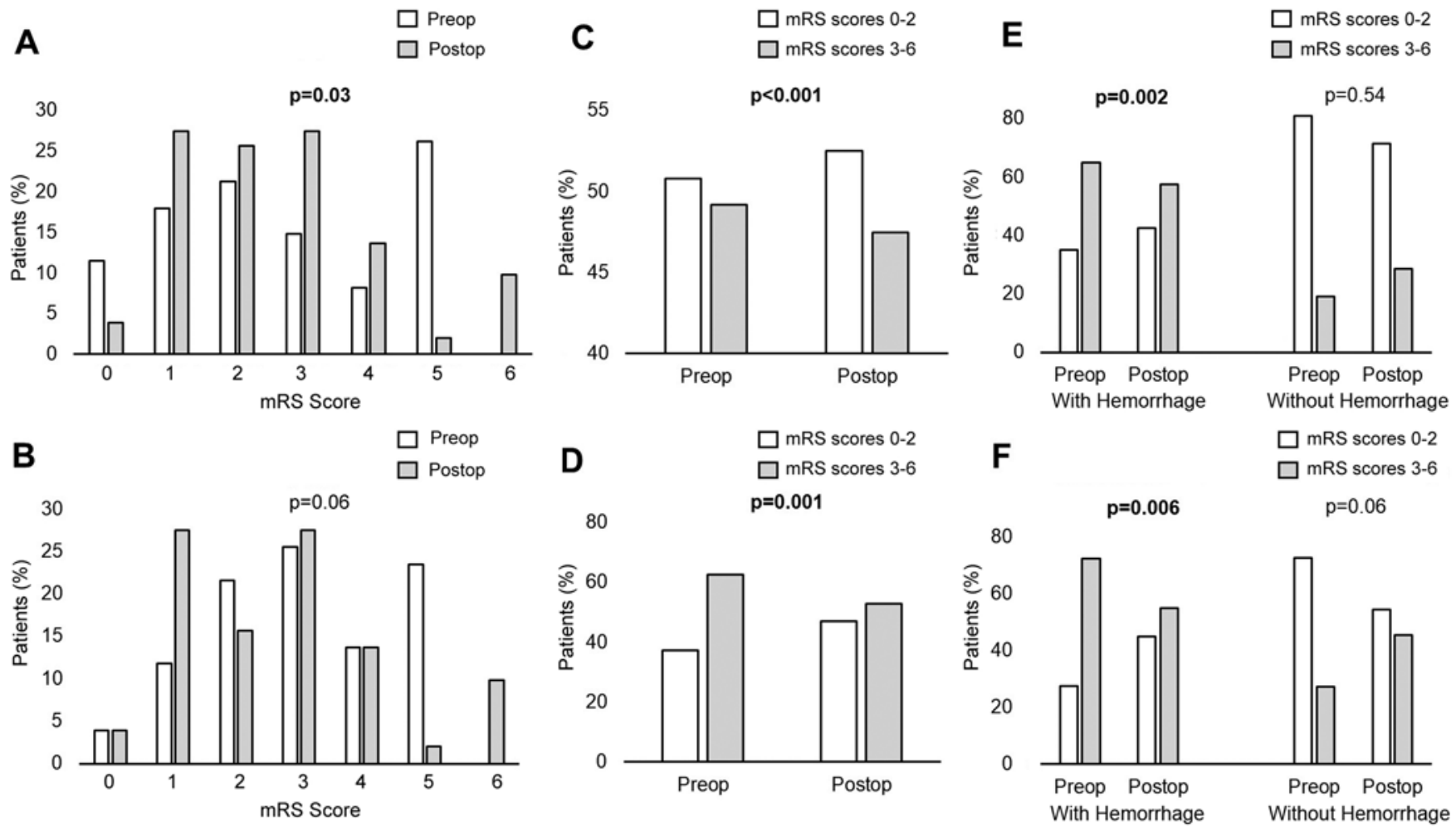

FIG. 2. A: Clinical status before and after surgery for patients 65-69 years of age (group $1 ; n=61$ ) measured with the mRS score. B: Preoperative and postoperative status for patients 70 years of age or older (group $2 ; n=51$ ) measured with the mRS score. C: Preoperative and postoperative status for group 1 showing the dichotomized mRS score (0-2 vs 3-6). D: Preoperative and postoperative status for group 2 showing the dichotomized mRS score. E: Preoperative and postoperative status for group 1 showing the dichotomized mRS score in relationship to ruptured AVM status (with hemorrhage vs without hemorrhage). F: Preoperative and postoperative status for group 2 showing the dichotomized mRS score in relationship to ruptured AVM status. 
favorable after AVM resection for both patients 65-69 years old and patients who are 70 years of age or older. Brain AVMs in elderly patients that were treated microsurgically were notable for small nidus size, high prevalence of AVM rupture, and low SM grades. Microsurgical resection is an important treatment modality for elderly patients with AVMs, and supp-SM grading is a useful tool for carefully selecting those patients who are most likely to achieve good neurological outcomes after resection.

\section{Acknowledgments}

We thank the staff of Neuroscience Publications at Barrow Neurological Institute for assistance with manuscript preparation.

\section{References}

1. Crawford PM, West CR, Chadwick DW, Shaw MD. Arteriovenous malformations of the brain: natural history in unoperated patients. J Neurol Neurosurg Psychiatry. 1986;49(1): $1-10$.

2. Friedlander RM. Clinical practice. Arteriovenous malformations of the brain. N Engl J Med. 2007;356(26):2704-2712.

3. Lawton MT, Rutledge WC, Kim H, et al. Brain arteriovenous malformations. Nat Rev Dis Primers. 2015;1:15008.

4. Mohr JP, Parides MK, Stapf C, et al. Medical management with or without interventional therapy for unruptured brain arteriovenous malformations (ARUBA): a multicentre, nonblinded, randomised trial. Lancet. 2014;383(9917):614-621.

5. Kim H, Abla AA, Nelson J, et al. Validation of the supplemented Spetzler-Martin grading system for brain arteriovenous malformations in a multicenter cohort of 1009 surgical patients. Neurosurgery. 2015;76(1):25-33.

6. Spetzler RF, Martin NA. A proposed grading system for arteriovenous malformations. J Neurosurg. 1986;65(4):476-483.

7. Pabaney AH, Reinard KA, Kole MK, et al. Management of arteriovenous malformations in the elderly: a single-center case series and analysis of outcomes. J Neurosurg. 2016; 125(1):145-151.

8. Burkhardt JK, Lasker GF, Winkler EA, et al. Microsurgical resection of brain arteriovenous malformations in the elderly: outcomes analysis and risk stratification. J Neurosurg. 2018; 129(5):1107-1113.

9. Lanzino G, Fergus AH, Jensen ME, et al. Long-term outcome after surgical excision of parenchymal arteriovenous malformations in patients over 60 years of age. Surg Neurol. 1997; 47(3):258-264.
10. Tong X, Wu J, Lin F, et al. Brain arteriovenous malformations in elderly patients: clinical features and treatment outcome. Acta Neurochir (Wien). 2015;157(10):1645-1654.

11. Kim H, Pourmohamad T, Westbroek EM, et al. Evaluating performance of the Spetzler-Martin supplemented model in selecting patients with brain arteriovenous malformation for surgery. Stroke. 2012;43(9):2497-2499.

12. Hashimoto H, Iida J, Kawaguchi S, Sakaki T. Clinical features and management of brain arteriovenous malformations in elderly patients. Acta Neurochir (Wien). 2004;146(10): 1091-1098.

13. Kim H, Al-Shahi Salman R, McCulloch CE, et al. Untreated brain arteriovenous malformation: patient-level meta-analysis of hemorrhage predictors. Neurology. 2014;83(7):590-597.

14. Neidert MC, Lawton MT, Kim LJ, et al. International multicentre validation of the arteriovenous malformation-related intracerebral haemorrhage (AVICH) score. J Neurol Neurosurg Psychiatry. 2018;89(11):1163-1166.

15. Ai X, Ye Z, Xu J, et al. The factors associated with hemorrhagic presentation in children with untreated brain arteriovenous malformation: a meta-analysis. J Neurosurg Pediatr. 2018;23(3):343-354.

\section{Disclosures}

Dr. Burkhardt: advisory board of Longeviti Neuro Solutions.

\section{Author Contributions}

Conception and design: Lawton, Burkhardt, Spetzler. Acquisition of data: Winkler, Catapano. Analysis and interpretation of data: Burkhardt, Winkler. Drafting the article: Burkhardt, Winkler. Critically revising the article: Lawton. Reviewed submitted version of manuscript: Lawton. Statistical analysis: Burkhardt, Winkler. Administrative/technical/material support: Burkhardt. Study supervision: Lawton, Spetzler.

\section{Correspondence}

Michael T. Lawton: c/o Neuroscience Publications, Barrow Neurological Institute, St. Joseph's Hospital and Medical Center, Phoenix, AZ.neuropub@barrowneuro.org. 